\title{
AAED1 modulates proliferation and glycolysis in gastric cancer
}

\author{
BEILI ZHANG, JUGANG WU, YANTAO CAI, MENG LUO, BING WANG and YAN GU \\ Department of General Surgery, Shanghai Ninth People's Hospital Affiliated to Shanghai Jiao Tong University \\ School of Medicine, Shanghai 200011, P.R. China
}

Received December 13, 2017; Accepted May 31, 2018

DOI: 10.3892/or.2018.6478

\begin{abstract}
Gastric cancer is one of the most common and lethal malignancies globally, especially in East Asia. Although significant progress has been made in the diagnosis and treatment of the disease, the overall survival rate remains unchanged at $20-25 \%$. Thus, there is an urgent need for a better understanding of the disease. Recent years have witnessed the critical roles of aberrant cancer cell metabolism in the maintenance of malignant properties of cancer cells, and targeting cancer cell metabolism has been regarded as a novel aspect in the development of treatments against cancer. In the present study, we identified a novel gene, AAEDl (AhpC/TSA antioxidant enzyme domain containing 1), which is upregulated in gastric cancer cells. By using lentivirus mediated transfection method, we silenced $A A E D I$ expression and silencing of $A A E D l$ inhibited cancer cell proliferation in vitro in gastric cancer cell lines, as demonstrated by cell viability and colony formation assay. Furthermore, we uncovered novel functions of $A A E D 1$ in regulating hypoxia inducible factor $1 \alpha$ (HIF1 $\alpha)$ and the resultant aerobic glycolysis, which was measured by extracellular flux analysis. Collectively, the results of the present study uncovered novel markers that could identify the possible molecular mechanisms involved in gastric cancer.
\end{abstract}

\section{Introduction}

Gastric cancer is one of the most common types of cancer worldwide and is ranked second in regards to cancer-related deaths worldwide (1). Although significant progress has been made in the diagnosis and treatment of gastric cancer, modest progress has been made in improving gastric cancer-related mortality. Furthermore, it has been hypothesized that the gastric cancer-related death rate will continue to increase in the future. Thus, there is an urgent need to identify novel

Correspondence to: Professor Yan Gu, Department of General Surgery, Shanghai Ninth People's Hospital, Shanghai Jiao Tong University School of Medicine, 639 Zhizaoju Road, Shanghai 200011, P.R. China

E-mail: yangu_surgeon@163.com

Key words: AAED1, gastric cancer, proliferation, glycolysis biomarkers that could be used to predict prognosis and elucidate the underlying molecular mechanisms $(2,3)$.

Ninety years ago, Otto Warburg published a series of studies linking metabolism and cancer through enhanced aerobic glycolysis (also known as the Warburg effect), which distinguishes cancer from normal tissues (4). According to the results of recent studies, aberrant cancer cell metabolism has been regarded as one of the hallmarks of cancer (5). It is known that solid tumors reside in a microenvironment with limited oxygen and nutrient supply; to survive under such a hostile microenvironment, tumor cells must shift their pattern of metabolism (6). Cancer cells are characterized by a glucose metabolism. Under hypoxic conditions, cancer cells shift their metabolism to glycolysis. Although, in terms of ATP generation, the process of glycolysis is not highly efficient because only two ATPs are generated. However, through glycolysis, cancer cells utilize glucose to form building blocks for macromolecule synthesis. Furthermore, lactate acid could be produced by glycolysis and accumulated lactic acid could cause an acidic microenvironment. The extracellular matrix becomes relatively unstable in an acidic environment, which facilitates the metastasis of cancer cells. Thus, it is hypothesized that targeting cancer cell metabolism may help improve and discover novel strategies against cancer (7).

Hypoxia inducible factor $1 \alpha(\mathrm{HIF} 1 \alpha)$ is regarded as a master regulator of aerobic glycolysis in cancer cells. HIF1 $\alpha$ initiates the transcription of genes that encodes transporters and enzymes in regulating glycolysis and the pentose phosphate pathway. The glycolytic products pyruvate and lactate could also induce HIF1 $\alpha$ accumulation, suggesting a feed-forward mechanism. The positive feedback loop further enhances the aerobic glycolysis cascade (8). Furthermore, tumor-promoting signaling pathways could also be activated under hypoxia, such as the PI3K/AKT/mTOR and MAPK signaling pathways. Activation of these pathways is also involved in HIF1 $\alpha$ and glycolysis upregulation (9). Another important role of glycolysis and HIF $1 \alpha$ is that HIF1 $\alpha$ transcribes many metastasis and angiogenesis genes. For example, metastasis-associated genes such as Twist and angiogenesis-related genes such as VEGF and VEGFR are all HIF1 $\alpha$ target genes. Additionally, the process of glycolysis also results in elevation of these genes, favoring metastasis. In recent years, the impact of cancer cell metabolism on gastric cancer has received an increasing amount of attention $(10,11)$. Glycolysis also participates in chemotherapy and radiotherapy resistance, which may account for the poor prognosis of gastric cancer patients (12). For 
example, under genotoxic stress conditions, tumor cells shift their glucose metabolism pattern to glycolysis through regulation of mitochondrial-localized metabolism regulator Sirtuin 4 (SIRT4) (13).

In conclusion, targeting cancer cell metabolism may be utilized to uncover novel predictive and treatment strategies for cancer. In the present study, using gastric cancer cells as a research model, we uncovered a novel protein AAED1 that was upregulated in gastric cancer. Furthermore, in vitro studies demonstrated that silencing of AAED1 inhibited the proliferation of gastric cancer cells. Mechanistic studies demonstrated that AAED1 positively regulated aerobic glycolysis via regulating HIF1 $\alpha$. The present study shed light on novel markers and strategies against gastric cancer and provide directions for further research.

\section{Materials and methods}

Cell culture. Human gastric cancer AGS and MGC-803 cell lines were used in the present study. The cell lines were obtained from the Cell Bank of Institute of Biochemistry and Cell Biology at the Chinese Academy of Sciences (Shanghai, China). The two cell lines were grown in Dulbecco's modified Eagle's medium (DMEM) supplemented with $10 \%$ fetal bovine serum (FBS) and 1\% penicillin-streptomycin (Gibco; Thermo Fisher Scientific, Inc., Waltham, MA, USA) at $37^{\circ} \mathrm{C}$ with humidified $5 \% \mathrm{CO}_{2}$

Establishment of AAED1-silenced cell lines. In order to silence AAED1 expression in gastric cancer cell lines, a lentivirus-mediated transfection method was used. A pLKO.1 TRC cloning vector that was obtained from Addgene Inc. (Cambridge, MA, USA) was used to express shRNA targets against AAED1 (14). The 21-bp targets against AAED1 were 5'-CGGCATTTCCTGTGTTACAT-3' and 5'-CGCGAT AGGAATAGGTTGGAT-3', respectively. The lentivirus was produced by co-transfecting AAED1-ilencing constructs with psPAX2 and pMD2.G vectors in a ratio of 4:3:1 into 293T cells. Stable AAED1-silenced cell lines were obtained by infecting AGS and MGC-803 cells and subsequent puromycin selection.

Cell viability assay. Cell Counting Kit-8 (CCK-8; Dojindo Molecular Technologies, Inc., Kumamoto, Japan) was used to measure the cell viability. Briefly, $200 \mu \mathrm{l}$ medium containing cells $(3,000 /$ well) was seeded into 96-well plates. After culturing for the indicated times, CCK-8 solution was added into each well at $37^{\circ} \mathrm{C}$. After $2 \mathrm{~h}$, the optical density values of each well were measured using a microplate reader at $450 \mathrm{~nm}$.

Colony-formation assay. AGS and MGC-803 cells $\left(5 \times 10^{2}\right)$ stably expressing shRNA targets against AAED1 and its relative control cells were seeded. After cultivating for 10 days, $4 \%$ paraformaldehyde was used to fix the cells followed by staining with $1 \%$ crystal violet. The colonies were counted subsequently. Briefly, the images of the plates were taken on a gel imager using a light filter, and colonies $>500 \mu \mathrm{m}$ or other appropriate diameters were counted.

Quantitative real-time PCR. Total RNA was extracted using TRIzol reagent (Invitrogen; Thermo Fisher Scientific, Inc.,
Waltham, MA, USA). Takara PrimeScript RT reagent kit was used for reverse transcription to obtain cDNA (Takara Bio, Inc., Otsu, Japan). The expression status of candidate genes and $\beta$-actin were determined by quantitative real-time PCR using an Applied Biosystems ${ }^{\circledR}$ 7900HT Real-Time PCR system (Thermo Fisher Scientific). $\beta$-actin was used as the reference genes. Thermocycling conditions were 40 cycles of $95^{\circ} \mathrm{C} 5 \mathrm{sec}$ and $60^{\circ} \mathrm{C} 30 \mathrm{sec}$. The method of quantifications were calculated according to a previous report (15). Primer sequences are listed in Table I.

Western blot analysis. Cells were washed with ice-cold phosphate-buffered saline (PBS) and lysed in RIPA buffer $(150 \mathrm{mM}$ $\mathrm{NaCl}, 1 \% \mathrm{NP}-40,50 \mathrm{mM}$ Tris/ $\mathrm{HCl}, \mathrm{pH} 8.0$ and $10 \%$ glycerol) containing protease and phosphatase inhibitors purchased from Selleck Chemicals LLC (Houston, TX, USA). Cell debris was removed by centrifugation at $12,000 \mathrm{rpm}$ for $20 \mathrm{~min}$ at $4^{\circ} \mathrm{C}$. Protein concentrations of the whole cell lysate were determined using a Thermo Pierce ${ }^{\circledR}$ BCA protein assay kit (Thermo Fisher Scientific). Equal amounts of $20 \mu \mathrm{g}$ total protein were subjected to SDS-PAGE separation and then transferred to polyvinylidene difluoride (PVDF) membranes. An antibody against AAED1 was purchased from Sigma-Aldrich (Merck KGaA). Glut1 (dilution factor 1:500; cat. no. 21829-1-AP), Hk2 (dilution factor 1:1,000; cat. no. 22029-1-AP), Ldha (dilution factor 1:3,000; cat. no. 19987-1-AP), Pgk1 (dilution factor 1:1,000; cat. no. 17811-1-AP), ERK1/2 (dilution factor 1:1,000; cat.no.16443-1-AP),Akt1 (dilution factor 1:1,000; 10176-2-AP), p-Akt1 (dlituion factor 1:1,000; cat. no. 66441-1-lg) and $\beta$-actin (dilution factor 1:3,000; cat. no. 60008-1-lg) antibodies were manufactured by Proteintech Group (Rosemont, IL, USA). Phospho-p44/p42 MAPK (Erk1/2) (Thr202/Tyr204) (dilution factor 1:1,000; cat. no. 9101) was purchased from Cell signaling Technology (Danvers, MA, USA).

Extracellular acidification rate (ECAR) and oxygen consumption rate $(O C R)$. Cellular glycolytic capacity and mitochondrial function were determined using the XF96 Extracellular Flux Analyzer (Seahorse Bioscience; Agilent Technologies, North Billerica, MA, USA), according to the manufacturer's instructions included in the Seahorse XF Glycolysis Stress Test kit (cat. no. 103020) and the Cell Mito Stress Test kit (cat. no. 103015). ECAR shows the ability of glycolysis. Before adding glucose, the ECAR value is known as the basic value, which reflects the acid production through the non-glycolytic pathway. When glucose is added, ECAR reflects the glycolysis capacity of the cells. When oligo is added, oxidative phosphorylation is inhibited, and the increased value of ECAR reflects the total glycolysis capacity of the cells, which is also known as the potential glycolysis ability of the cells. The total numerical value is the maximum glycolysis ability of the cells. 2-DG, which is finally added, is an inhibitor of glycolysis, and the ECAR change after this step reflects the acid production through the non-glycolytic pathway. In OCR assay, the basal value before adding oligo reflects the basic oxygen consumption of the cells, which includes two components; mitochondrial oxidative phosphorylation and proton leakage oxygen consumption. Protons form electrical potential energy on the mitochondrial membrane via the respiratory chain. Some of protons reflux and ATP 
Table I. Primer sequences used in the present study.

\begin{tabular}{ll}
\hline AAED1 forward & $\begin{array}{l}\text { 5'-AAGGAGACCCAGCTCAGCAAG } \\
\text { GTG-3' }\end{array}$ \\
AAED1 reverse & $\begin{array}{l}\text { 5'-AGCAGGTTAGACACTGCACGA } \\
\text { GGA-3' }\end{array}$ \\
GLUT1 forward & 5'-CTTTGTGGCCTTCTTTGAAGT-3' \\
GLUT1 reverse & 5'-CCACACAGTTGCTCCACAT-3' \\
HK2 forward & 5'-GATTGTCCGTAACATTCTCATC \\
& GA-3' \\
HK2 reverse & 5'-TGTCTTGAGCCGCTCTGAGAT-3' \\
PGK1 forward & 5'-CAAGGTTAAAGCCGAGCCAGCC \\
& AA-3' \\
PGK1 reverse & 5'-GCCTTCTGTGGCAGATTGACT \\
& CC-3' \\
LDHA forward & 5'-TGGAGATTCCAGTGTGCCTGTAT \\
& GG-3' \\
LDHA reverse & 5'-CACCTCATAAGCACTCTCAACCA \\
& CC-3' \\
$\beta$-actin forward & 5'-CCAACCGCGAGAAGATGACC \\
& CA-3' \\
$\beta$-actin reverse & 5'-ATCACGATGCCAGTGGTACG-3' \\
\hline
\end{tabular}

AAED1, AhpC/TSA antioxidant enzyme domain containing 1; GLUT1, glucose transporter 1; HK2, hexokinase 2; PGK1, phosphoglycerate kinase 1; LDHA, lactate dehydrogenase A.

is formed through ATP synthetase, and the potential energy is transformed into energy in the form of ATP. Another part of the proton is oxidized by the proton in the mitochondrial membrane, and the potential energy is not used to synthesize ATP, but to heat energy. After the addition of ATP synthase inhibitor oligo, the decrease in oxygen consumption reflects the oxygen consumption of the body in the process of ATP synthesis, which indirectly reflects the ATP production of the cells. FCCP is a kind of uncoupler that can be regarded as a proton carrier, which allows a large number of protons to flow back. Oxygen consumption after the addition of FCCP reflects the largest mitochondrial oxygen consumption capacity, indirectly reflecting the maximum breathing capacity. Finally, two types of agents, amphoterin A and rotenone, were added, and they were respiratory chain inhibitors, which completely prevented the oxygen consumption of mitochondria.

Immunohistochemistry. The 80 clinical tissue samples used in this study were histopathologically and clinically diagnosed at the Shanghai Ninth People's Hospital Affiliated to Shanghai Jiaotong University School of Medicine (Shanghai, China) between June 2014 and May 2017. The age distribution of patients ( 47 males and 33 females) was between 28 and 75 years. Prior patient consent and approval from the Institutional Research Ethics Committee were obtained. Paraffin-embedded tissue slides were deparaffinized in xylene, rehydrated through a graded alcohol series, blocked in methanol containing $3 \%$ hydrogen peroxide, and then incubated with an AAED1 antibody (dilution 1:50; cat. no. HPA021294; Sigma-Aldrich, Merck KGaA). Followed by PBS solution rinsing, the slides were incubated with secondary antibodies and peroxidase reagent at room temperature (cat. no. GK6007; Beijing Genetech, Co., Ltd., China). At the end, the slides were incubated with 3,3'-diaminobenzidine solution at room temperature for $10 \mathrm{~min}$ and counterstained with hematoxylin. A scoring scale was used to evaluate the percentage of cells stained $(0,<10 \% ; 1,10-25 \% ; 2,>25-50 \% ; 3,>50-75 \%$; and $4,>75 \%)$ and intensity of staining ( 0 , negative; 1 , low; 2 , moderate; and 3 , strong). The overall staining scores were determined by combining the two scores (frequency plus intensity). An immunohistochemical score $>6$ was defined as high expression, whereas a score $\leq 6$ was defined as low expression (16).

Promoter activity assessment by Dual-Luciferase assay. AGS and MGC-803 cells were seeded on 96-well culture plates and transfected with indicated vectors using Invitrogen ${ }^{\mathrm{TM}}$ Lipofectamine $^{\mathrm{TM}} 2000$ (Thermo Fisher Scientific). HIF1 $\alpha$ activity was assessed using HRE-luciferase firefly luciferase construct (Addgene plasmid 26731) (17). pRL-TK (Promega, Madison, WI, USA) was used as an internal control. Firefly and Renilla luciferase activities were measured using a Dual-Luciferase system (Promega), as described in the manufacturer's protocol.

Measurement of intracellular ROS levels. Intracellular ROS were detected using a Reactive Oxygen Species Assay kit (Beyotime Institute of Biotechnology, Haimen, China). In brief, cells were washed with PBS. Then, cells were stained with $10 \mu \mathrm{mol} / 1 \mathrm{DCFH}-\mathrm{DA}$ at $37^{\circ} \mathrm{C}$ for $20 \mathrm{~min}$ according to the manufacturer's instructions. DCFH-DA was deacetylated intracellularly by non-specific esterase, which was further oxidized by ROS to the fluorescent compound 2,7-dichlorofluorescein (DCF). DCF fluorescence was detected using a FACScan flow cytometer (Becton-Dickinson; BD Biosciences, Franklin Lakes, NJ, USA).

Statistical analysis. All data are presented as the mean \pm SD; experiments were repeated at least three times. Two-tailed unpaired Student's t-tests and one-way analysis of variance (ANOVA) and post hoc tests were used to evaluate the data. SPSS version 16.0 (SPSS, Inc., Chicago, IL, USA) was used for data analysis. Differences were considered significant at ${ }^{*} \mathrm{P}<0.05,{ }^{* * *} \mathrm{P}<0.01,{ }^{* * *} \mathrm{P}<0.001,{ }^{* * * * *} \mathrm{P}<0.0001$ as indicated in the figures.

\section{Results}

AAEDI expression is upregulated in gastric cancer. To verify the expression status of AAED1 in gastric cancer, IHC staining was performed using an AAED1 antibody. As shown, AAED1 was significantly higher in gastric tumor samples than that in normal paratumor samples (Fig. 1A). The scoring criteria was listed as negative, weak, modest and strong (Fig. 1B). Results indicated that AAED1 expression was significantly higher in gastric tumor samples than that in the paratumor samples (Fig. 1C). Taken together, these results suggested that AAED1 may be a tumor-promoting marker in gastric cancer.

AAED1 regulates the proliferation of gastric cancer cells. To confirm the role of AAED1 in gastric cancer cell proliferation, 
A

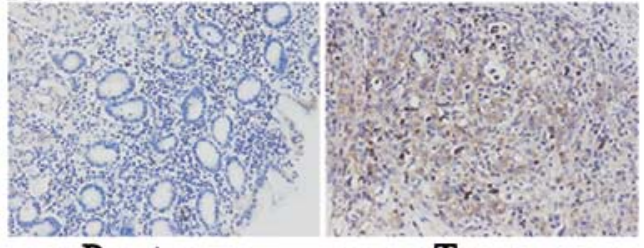

Paratumor

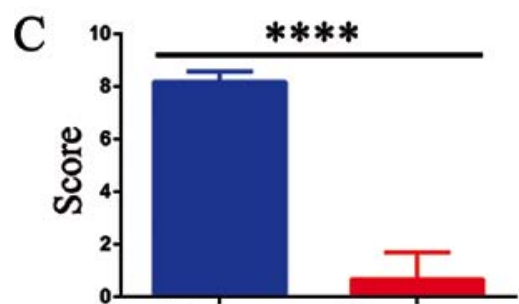

B

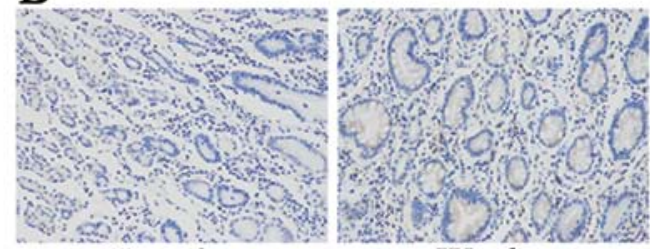

Negative

Weak

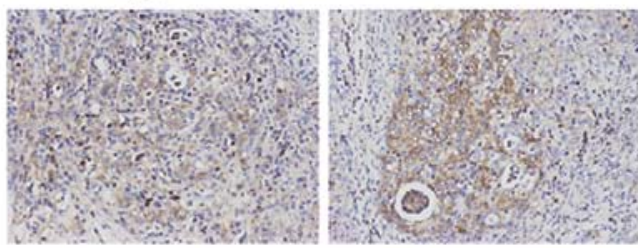

Modest

Strong

\section{Tumor Paratumor}

Figure 1. AAED1 expression ia upregulated in gastric cancer. (A) Expression of AAED1 was higher in tumor samples than that in paratumor samples. (B) The scoring parameter of AAED1 expression in patients with gastric cancer. (C) The expression of AAED1 was significantly higher in tumor samples than that in paratumor samples from patients with gastric cancer. AAED1, AhpC/TSA antioxidant enzyme domain containing $1{ }^{* * * * *} \mathrm{P}<0.0001$.
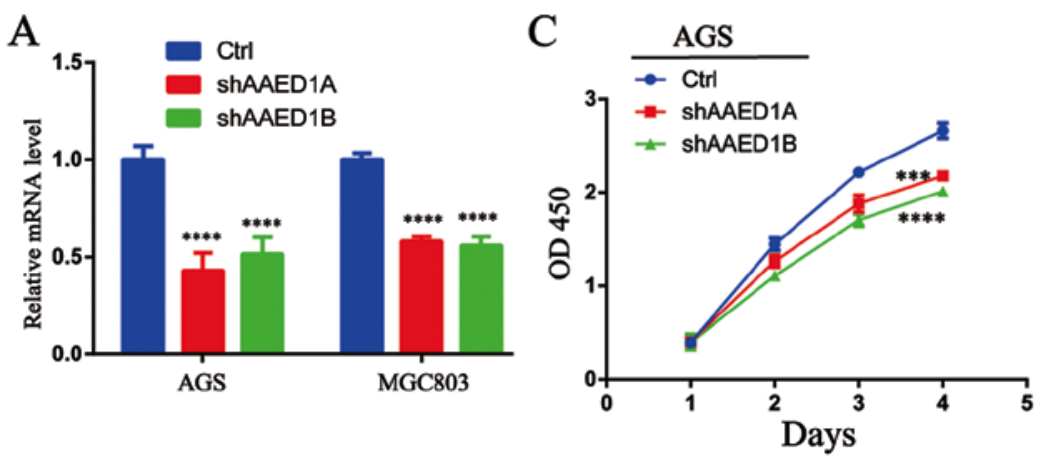

B

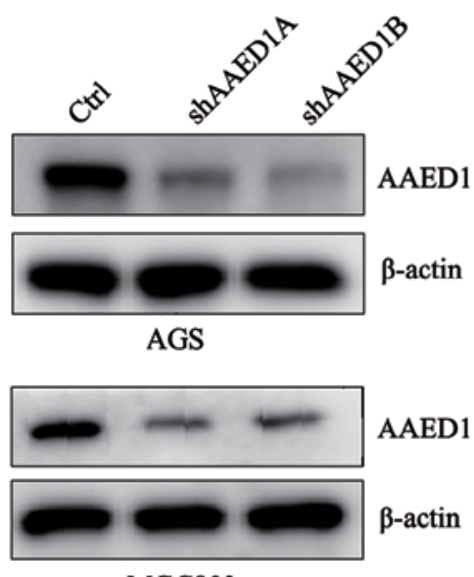

D

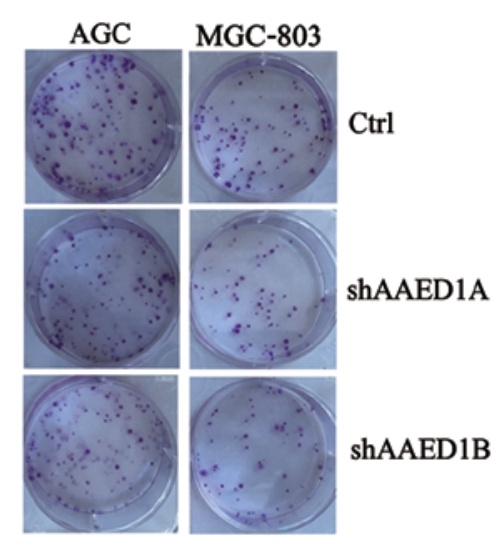

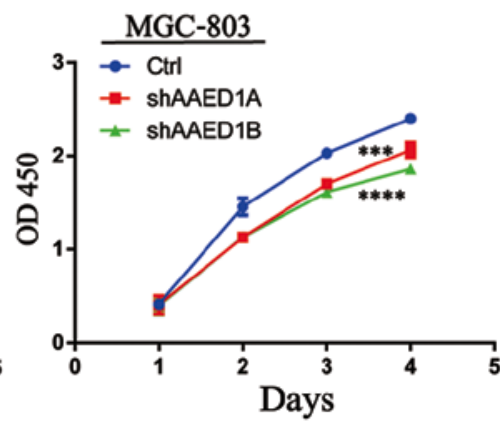

E
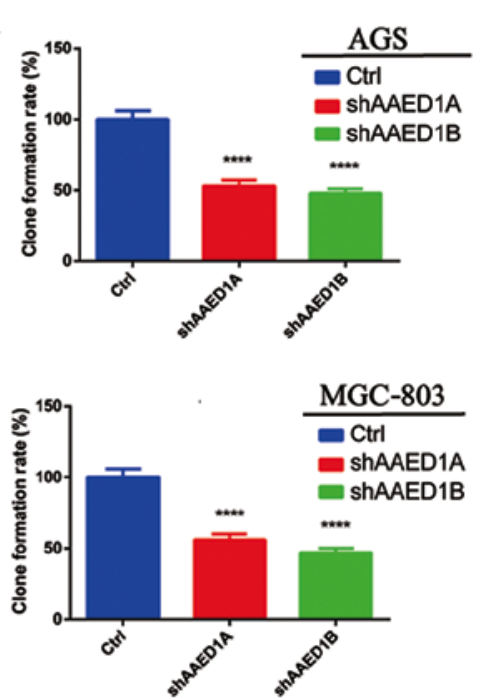

Figure 2. AAED1 modulates the proliferation of gastric cancer cells. (A) Quantitative real-time PCR assay validated the silencing efficacy of AAED1 in AGS and MGC-803 cells. (B) The knockdown efficiency was further supported by western blot analysis with an AAED1 antibody. (C) Silencing of AAED1 inhibited viability of AGS and MGC-803 cells as demonstrated by CCK- 8 assay. (D and E) Decreased expression of AAED1 inhibited colony formation capacity of AGS and MGC-803 cells. ${ }^{* * *} \mathrm{P}<0.001 .{ }^{* * * *} \mathrm{P}<0.0001$. AAED1, AhpC/TSA antioxidant enzyme domain containing 1.

we firstly silenced AAED1 expression in gastric cancer cell lines using a lentivirus-mediated method. Two shRNA targets that efficiently suppressed AAED1 mRNA expression in AGS and MGC-803 cells were generated (Fig. 2A). Immunoblotting with AAED1 antibodies further verified the silencing effect (Fig. 2B). Next, a CCK-8 cell viability assay was 

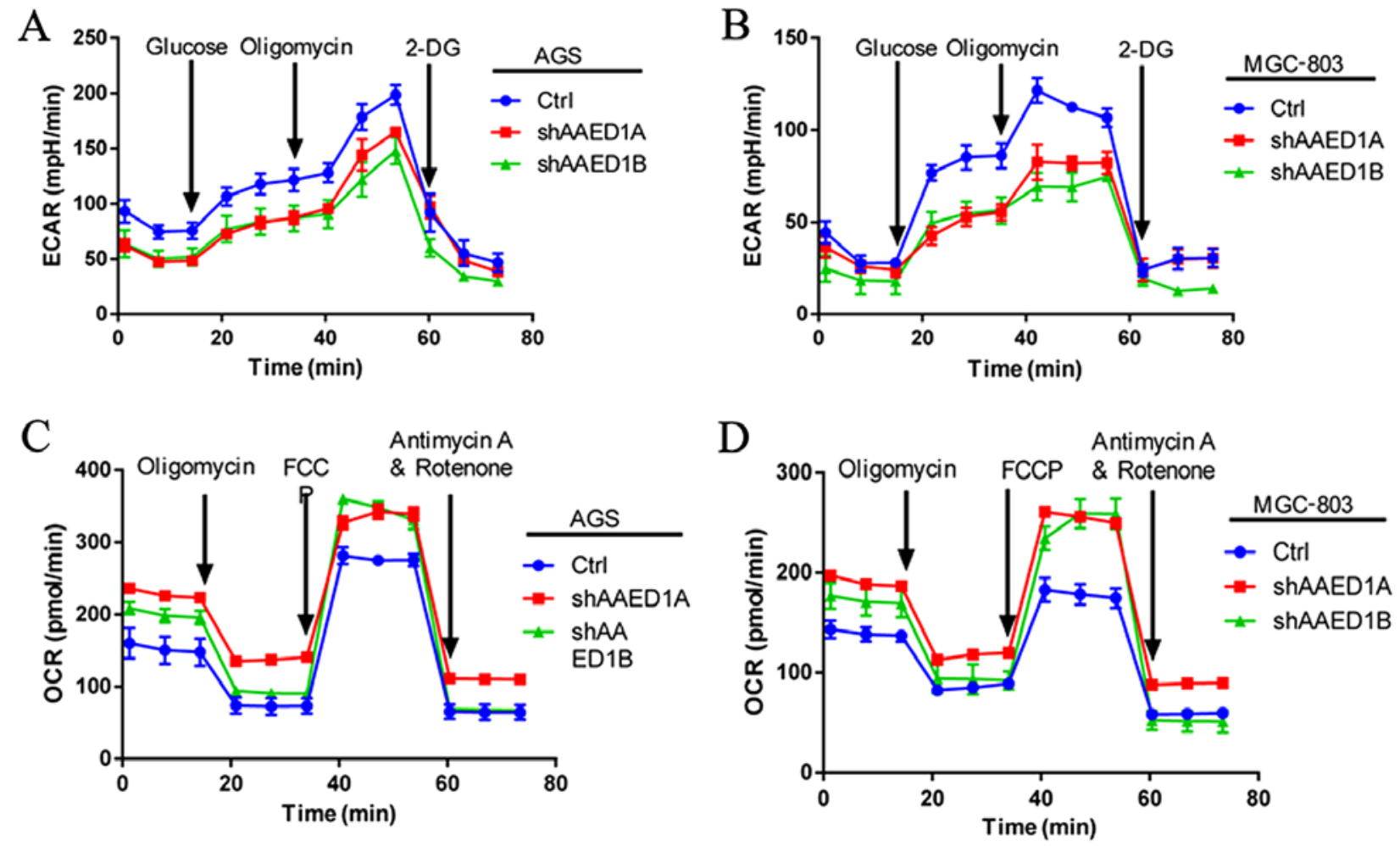

Figure 3. AAED1 positively regulates aerobic glycolysis in gastric cancer cells. (A and B) AAED1 knockdown inhibited ECAR levels in AGS and MGC-803 cells, suggesting that AAED1 positively regulated glycolysis of gastric cancer cells. (C and D) AAED1 silencing increased OCR levels, indicating AAED1 as a negative regulator of mitochondrial respiration. AAED1, AhpC/TSA antioxidant enzyme domain containing 1; ECAR, extracellular acidification rate; OCR, oxygen consumption rate.

performed, and the results indicated that silencing of AAED1 expression inhibited cell viability in the AGS and MGC-803 cells (Fig. 2C). Finally, the impact of AAED1 on colony formation capacity was assessed. As shown, silencing of AAED1 expression significantly inhibited colony formation capacity (Fig. 2D and E). Collectively, these results suggested that AAED1 may have positively regulated proliferation in gastric cancer cells.

AAED1 positively regulates aerobic glycolysis in gastric cancer cells. Aberrant cancer cell metabolism is pronounced in cancer cells to facilitate uncontrolled proliferation. Aerobic glycolysis is the best characterized metabolic reprogramming that regulates cancer cell proliferation. Thus, we analyzed the impact of AAED1 on gastric cancer cell metabolism. Through glycolysis, cancer cells utilize glucose to produce lactate and increased levels of lactate could produce an acidic environment that could be measured by extracellular acidification rate (ECAR). Our ECAR measurement results demonstrated that silencing of AAED1 expression decreased ECAR levels, indicating that AAED1 is a positive regulator of aerobic glycolysis (Fig. 3A and B). During glycolysis, mitochondrial respiration was impaired and was measured using the oxygen consumption rate (OCR). In AAED1-silenced AGS and MGC-803 cells, we observed an increase in OCR levels, which further reinforced the impact of AAED1 on aerobic glycolysis (Fig. 3C and D). In conclusion, our results suggested that AAED1 is a positive regulator of glycolysis, and AAED1 supports the proliferation of gastric cancer cells.
AAEDI positively regulates the ERK/Aktl/HIFla signaling pathway in gastric cancer cells. To search for the molecular pathway that participates in aerobic glycolysis by AAED1 in gastric cancer cells, the changes in HIFl $\alpha$ expression in AAED1-knockdown AGS and MGC-803 cells were examined. As observed, HIFl $\alpha$ protein levels were observably decreased in the AAED1-silenced cells. Activation of ERK1/2 and Akt1 has been reported to positively regulate aerobic glycolysis and to regulate HIF1 $\alpha$ and HIF1 $\alpha$-transcriptional activity. Thus, we performed immunoblot analysis to observe changes in the ERK1/1 and Akt1 activation status in the AGS and MGC-803 cells. As shown, silencing of AAED1 inhibited the activation of ERK1/2 and Akt1, which may account for the regulation of HIF1 $\alpha$ by AAED1 in gastric cancer cells (Fig. 4).

AAED1 regulates ROS generation in gastric cancer cells. AAED1 may play an antioxidant role in cancer cells. As observed above, AAED1 regulated ERK1/2 and Akt1 activation, two signaling pathways that may regulate ROS generation and antioxidant response. Thus, we measured the influence of AAED1 on ROS generation in AGS and MGC-803 cells. As shown, silencing of AAED1 expression in these two gastric cancer cell lines decreased intracellular ROS levels (Fig. 5A and B).

AAED1 modulates the expression of glycolysis-related genes and HIFla transcriptional activity. Aerobic glycolysis is a multiple step enzymatic process to metabolize glucose. Among the many glycolysis genes, GLUT1, HK2, PGK1 and 


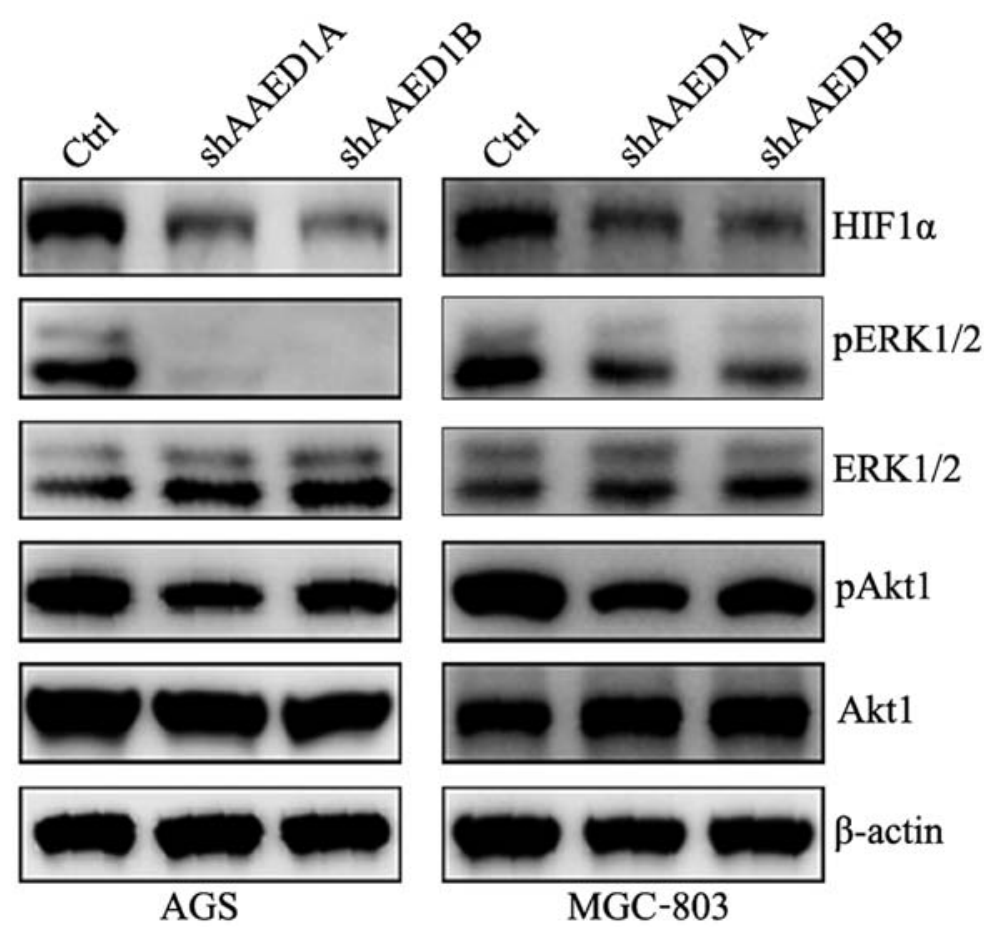

Figure 4. AAED1 positively regulates the ERK/Akt1/HIF1 $\alpha$ signaling pathway in gastric cancer cells. AAED1 knockdown decreased the activation status of ERK1/2 and Akt1 in AGS and MGC-803 cells. Furthermore, the expression status of HIF1 $\alpha$ was decreased in AAED1-knockdown cells, indicating that AAED1 may regulate the ERK/Akt1/HIF1 $\alpha$ axis in gastric cancer cells. AAED1, AhpC/TSA antioxidant enzyme domain containing 1.
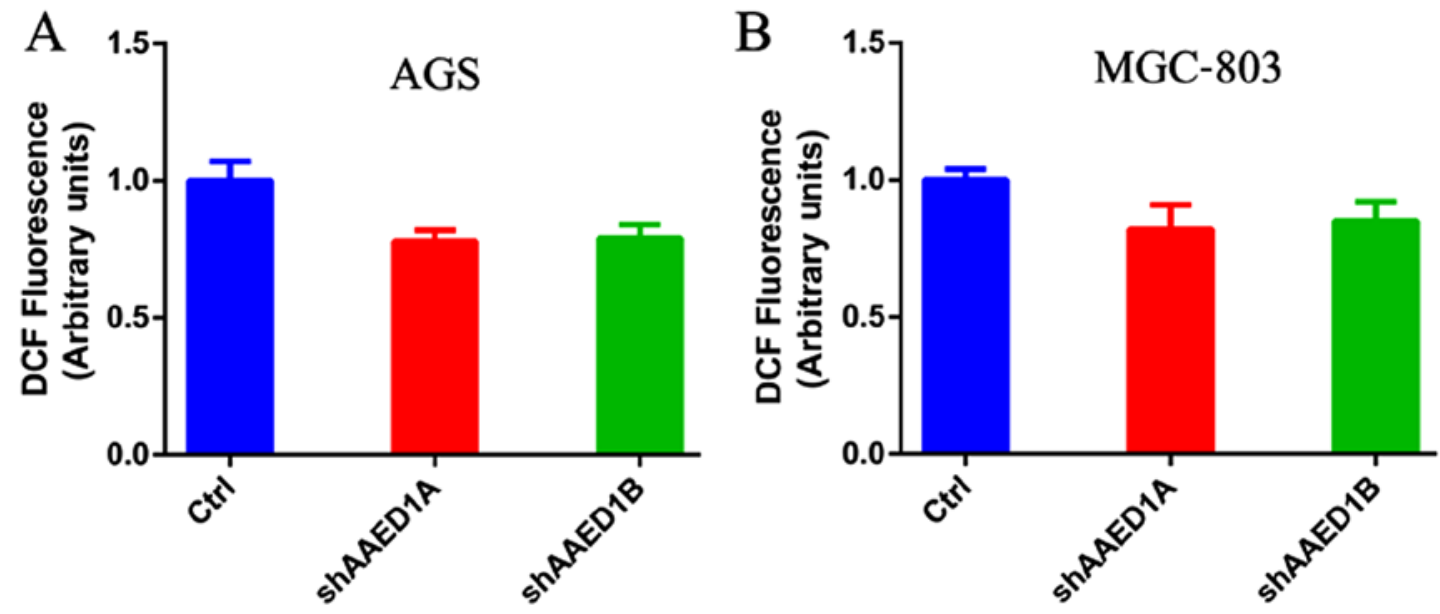

Figure 5. AAED1 controls the levels of intracellular ROS. ROS production has been demonstrated to regulate aerobic glycolysis, HIF1 $\alpha$ stability and ERK1/2 activation. (A and B) In AAED1-silenced AGS and MGC-803 cells, we observed a decrease in intracellular ROS levels. AAED1, AhpC/TSA antioxidant enzyme domain containing 1 ; ROS, reactive oxygen species.

LDHA are reported to catalyze the rate-limiting steps and are direct HIF1 $\alpha$ target genes. Thus, quantitative real-time PCR (qPCR) analysis was performed to assess the impact of AAED1 on the expression status of GLUT1, HK2, PGK1 and LDHA. As shown, AAED1 knockdown significantly decreased the expression of these HIF1 $\alpha$-targeted, glycolysis genes (Fig. 6A-D). Furthermore, western blot analysis results were consistent with the qPCR results, and the protein levels of GLUT1, HK2, PGK1 and LDHA were decreased in the presence of AAED1 knockdown (Fig. 6E and F). GLUT1, HK2, PGK1 and LDHA are HIF1 $\alpha$ target genes and conserve hypoxia response elements on their promoter region.
HIF1 $\alpha$ transcription activity could be assessed by using HRE-luciferase activity. Then, we performed a Dual-Luciferase assay, and our results demonstrated that AAED1 significantly regulated HRE-luciferase activity in a dose-dependent manner (Fig. 6G and $\mathrm{H}$ ).

In conclusion, the results of the present study demonstrated that AAED1 levels were increased in patients with gastric cancer. In vitro results suggested that AAED1 positively regulated ERK1/2 and AKT1 activation and the relevant HIF1 $\alpha$ upregulation. Increased HIF1 $\alpha$ resulted in increased expression of glycolysis genes and enhanced glycolysis, which favors uncontrolled proliferation of gastric cancer cells (Fig. 7). 
A

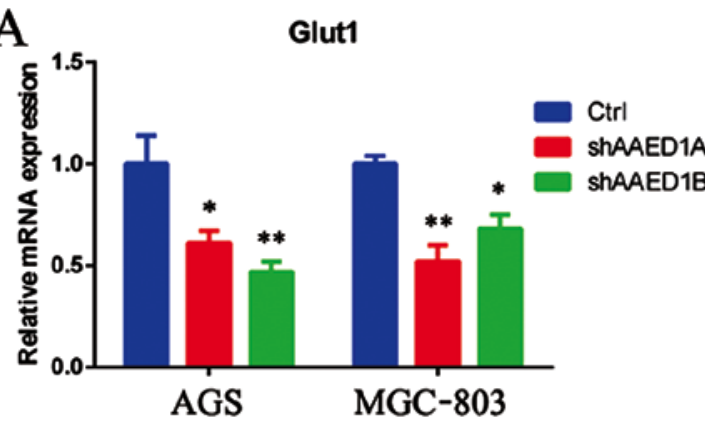

C

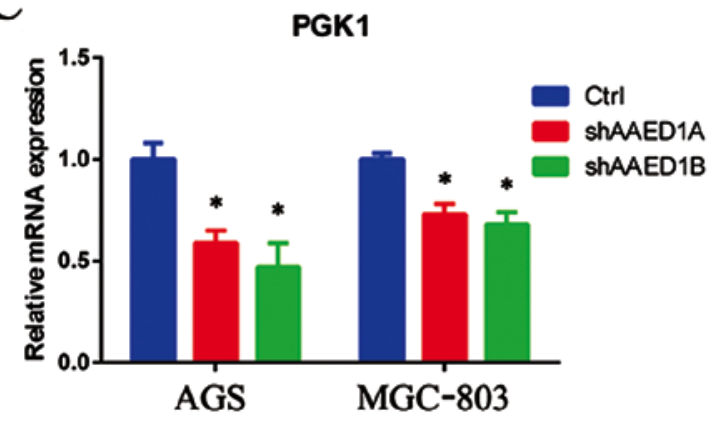

$\mathrm{E}$
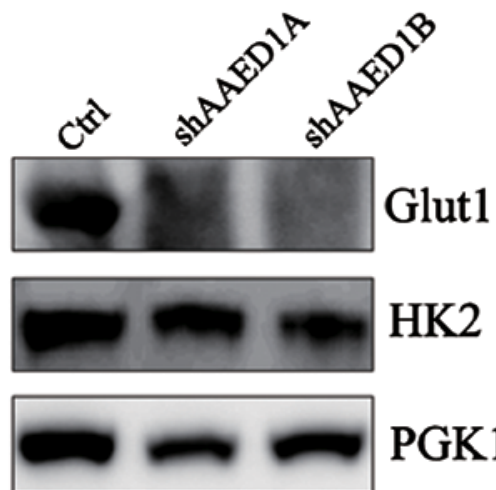

\section{PGK1}

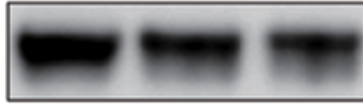

LDHA

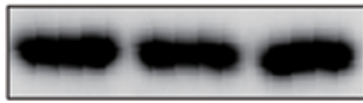

$\beta$-actin

AGS

$\mathrm{G}$

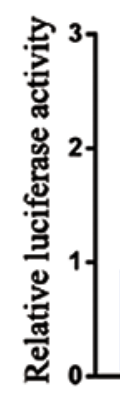

HRE-Luciferase (ng) 100

AAED1 (ng) 0

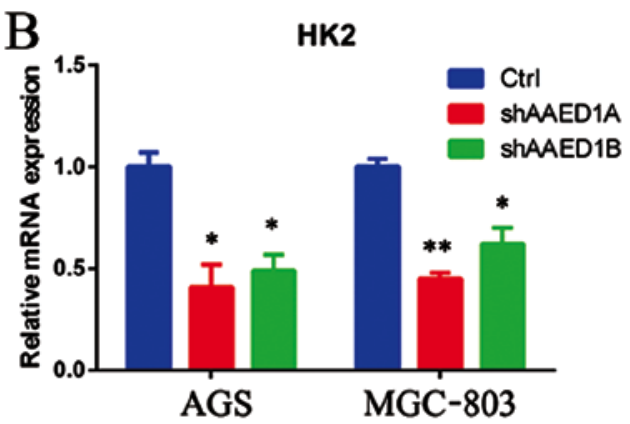

D

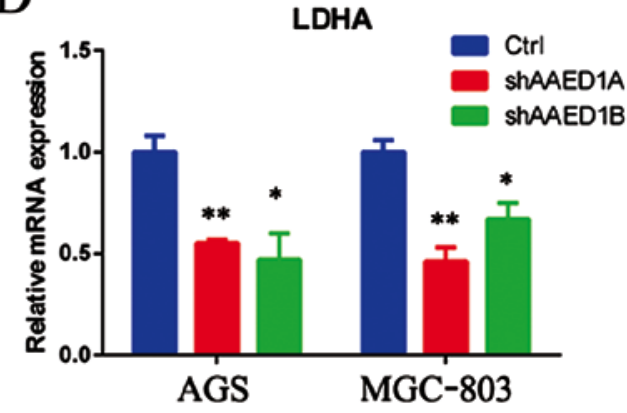

F
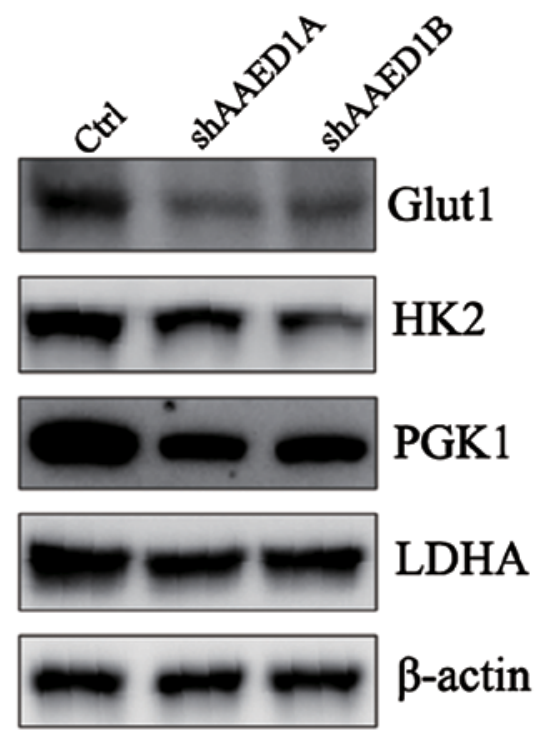

MGC803

$\mathrm{H}$

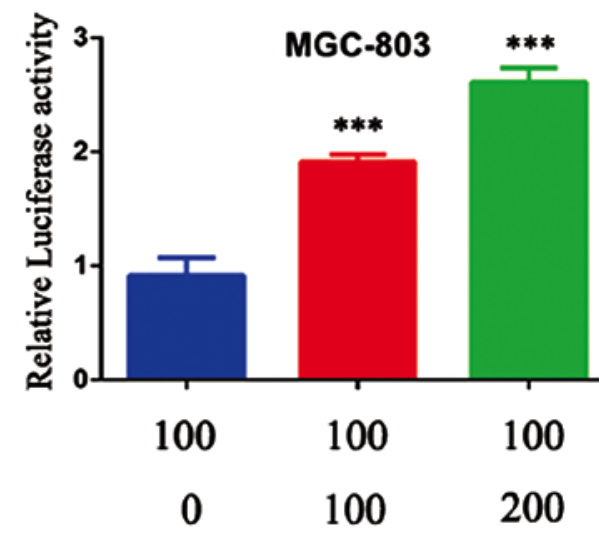

Figure 6. AAED1 modulates the expression of glycolysis genes and HIF1 $\alpha$ transcriptional activity. Aerobic glycolysis is a multi-step process catalyzed by glycolysis enzymes. (A-D) In AAED1-silenced AGS and MGC-803 cells, we observed a significant decrease in mRNA levels of Glut1, HK2, PGK1 and LDHA, all of which are HIF1 $\alpha$ targets. (E and F) We observed a relevant decrease in the protein levels of these enzymes. (G and $\mathrm{H}$ ) Additionally, we observed the impact of AAED1 on HIF1 $\alpha$ transcriptional activity by HRE-luciferase assay and the results demonstrated that AAED1 regulated HRE-luciferase activity in a dose-dependent manner. ${ }^{*} \mathrm{P}<0.05,{ }^{* *} \mathrm{P}<0.01,{ }^{* * *} \mathrm{P}<0.001$. AAED1, AhpC/TSA antioxidant enzyme domain containing 1; Glut1, glucose transporter 1; HK2, hexokinase 2; PGK1, phosphoglycerate kinase 1; LDHA, lactate dehydrogenase A. 


\section{AAED1}

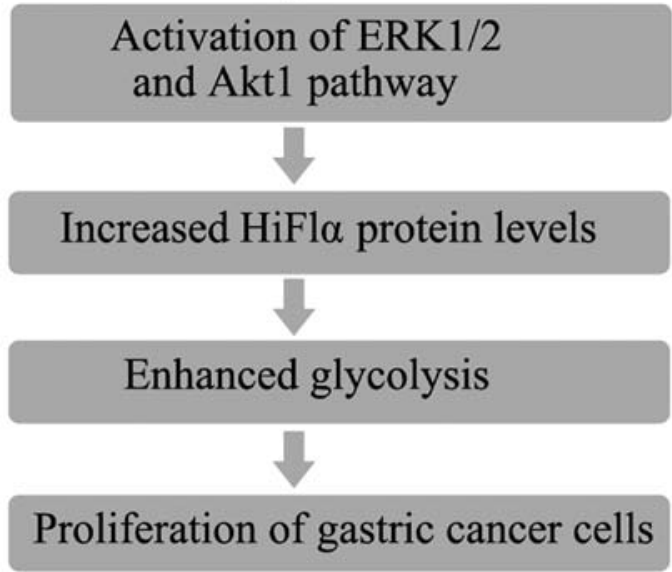

Figure 7. Working model of the study. In the present study, we demonstrated that AAED1 was elevated in patients with gastric cancer. Mechanistic studies demonstrated that AAED1 regulated the ERK/Akt1/HIF1 $\alpha$ axis, thereby enhancing glycolysis, which supported the uncontrolled proliferation of gastric cancer cells. AAED1, AhpC/TSA antioxidant enzyme domain containing 1 ; HIF1 $\alpha$, hypoxia inducible factor $1 \alpha$ subunit.

\section{Discussion}

In the present study, we demonstrated that AAED1 was upregulated in gastric cancer. In vitro studies demonstrated that AAED1 regulated cell proliferation. Mechanistic studies demonstrated that AAED1 regulated HIF1 $\alpha$ and HIF1 $\alpha$-transcriptional activity, leading to enhanced glycolysis in gastric cancer cells.

AAED1 consists of the AphC/TSA antioxidant enzyme domain, which indicates that it may play certain roles in antioxidant response. Thus, in the present study, we measured its effect on ROS generation in gastric cancer cells. Our results demonstrated that silencing of AAED1 in AGS and MGC-803 cells inhibited intracellular ROS levels. Previous studies have demonstrated that intracellular ROS levels regulate HIF1 $\alpha$ protein stability, resulting in metabolism reprogramming $(18,19)$. Consistently, we also demonstrated that HIF1 $\alpha$ protein levels were significantly decreased in AAED1-silenced gastric cancer cells. Further studies are needed to examine the impact of AAED1 on HIF1 $\alpha$ protein level stability regulation, especially post-translational modifications, such as ubiquitination, as intracellular ROS levels were found to regulate hydroxylation of HIF1 $\alpha$, leading to ubiquitination of this protein (20-22). Another possible impact of AAED1 on HIF1 $\alpha$ protein level regulation is that AAED1 regulated the activation status of ERK1/2 and Akt1 $(23,24)$. Phosphorylation of HIF $1 \alpha$ by ERK $1 / 2$ is also critical for the stability of HIF $1 \alpha$, as phosphorylation of HIF $1 \alpha$ and subsequent ubiquitination of HIF1 $\alpha$ by FBW7 could lead to destruction of HIF1 $\alpha$ (25). Another possible mechanism underlying AAED1 in intracellular ROS generation might be attributed to nuclear factor (erythroid-derived 2)-like 2 (NRF2)-mediated antioxidant responses. NRF2 has been demonstrated in many type of cancers, such as breast cancer, liver cancer and pancreatic cancer (26-29). Aberrancies in intracellular ROS levels were found to regulate NRF2 protein levels and NRF2-mediated antioxidant response, leading to cell proliferation and chemotherapy and radiotherapy resistance (30). Thus, the present study indicated further directions for studying the impact of AAED1 on NRF2 and on chemotherapy and radiotherapy resistance, two facets that may regulate a poorer survival in patients with gastric cancer.

In the present study, we demonstrated that AAED1 regulated aerobic glycolysis in gastric cancer cells. The impact of aerobic glycolysis has been demonstrated to play vital roles in maintaining cancer cell proliferation under severely hypoxic conditions caused by limited oxygen and nutrient supply. Furthermore, glycolysis produces lactate, leading to an acidic microenvironment of cancer cells (31). Under acidic conditions, the extracellular matrix becomes unstable, which favors the metastasis of cancer cells $(32,33)$. For example, in gastric cancer cells, epithelial-mesenchymal-transition (EMT) regulator Snail-mediated repression of glycolytic enzyme fructose-bisphosphatase 1 (FBP1) provides metastatic advantages to gastric cancer cells (34). Some EMT regulators such as Twist1, are also HIF1 $\alpha$ targets. Moreover, glycolysis regulator HIF1 $\alpha$ could also regulate angiogenesis, which plays significant roles in the metastasis of cancerous cells $(35,36)$. Thus, the present observation inspired us to carry out further studies to examine the impact of AAED1 and AAED1-mediated glycolysis on the metastasis of gastric cancer.

AAED1 was observed in the present study to maintain proliferation and glycolysis of gastric cancer, indicating its possible roles in cancer development. In line with this, we demonstrated that AAED1 protein levels were significantly higher in gastric cancer tissues than the relevant adjacent tissues, indicating its roles as a possible marker for the diagnosis and prognosis of gastric cancer. In our future studies, we plan to increase the number of patients and perform IHC staining and overall survival analysis to validate the possible role of AAED1 in the prognosis of gastric cancer. Furthermore, based on the role of AAED1 on gastric cancer proliferation, identifying small molecules that could inhibit AAED1 activity may help to uncover novel strategies for the treatment of gastric cancer.

In conclusion, the present study provides a novel role for AAED1 in gastric cancer cell proliferation and metabolic regulation. These results suggested that AAED1 may be a promising therapeutic target for gastric cancer.

\section{Acknowledgements}

We acknowlege Henghua Zhou for her help in pathological assistance.

\section{Funding}

The present study was supported by the Shanghai Municipal Commission of Health and Family Planning (grant no. 20164 Y0255).

\section{Availability of data and materials}

The datasets used during the present study are available from the corresponding author upon reasonable request. 


\section{Authors' contributions}

BZ performed most of the assays in the manuscript. JW, YC, ML and BW collected and analysed the data; YG designed and supervised the manuscript. All authors read and approved the manuscript and agree to be accountable for all aspects of the research in ensuring that the accuracy or integrity of any part of the work are appropriately investigated and resolved.

\section{Ethics approval and consent to participate}

The study protocol was approved by the Bioethical Commission of Shanghai Ninth People's Hospital Affiliated to Shanghai Jiaotong University School of Medicine.

\section{Patient consent for publication}

Not applicable.

\section{Competing interests}

The authors state that they have no competing interests.

\section{References}

1. Siegel RL, Miller KD and Jemal A: Cancer statistics, 2017. CA Cancer J Clin 67: 7-30, 2017.

2. Fu DG: Epigenetic alterations in gastric cancer (Review). Mol Med Rep 12: 3223-3230, 2015.

3. Ushijima T and Sasako M: Focus on gastric cancer. Cancer Cell 5: 121-125, 2004.

4. Koppenol WH, Bounds PL and Dang CV: Otto Warburg's contributions to current concepts of cancer metabolism. Nat Rev Cancer 11: 325-337, 2011.

5. Hanahan D and Weinberg RA: Hallmarks of cancer: The next generation. Cell 144: 646-674, 2011.

6. Keith B, Johnson RS and Simon MC: HIF1 $\alpha$ and HIF2 $\alpha$ : Sibling rivalry in hypoxic tumour growth and progression. Nat Rev Cancer 12: 9-22, 2011.

7. Tennant DA, Durán RV and Gottlieb E: Targeting metabolic transformation for cancer therapy. Nat Rev Cancer 10: 267-277, 2010.

8. Denko NC: Hypoxia, HIF1 and glucose metabolism in the solid tumour. Nat Rev Cancer 8: 705-713, 2008.

9. Majmundar AJ, Wong WJ and Simon MC: Hypoxia-inducible factors and the response to hypoxic stress. Mol Cell 40: 294-309, 2010.

10. Rankin EB and Giaccia AJ: The role of hypoxia-inducible factors in tumorigenesis. Cell Death Differ 15: 678-685, 2008.

11. Xiao S and Zhou L: Gastric cancer: Metabolic and metabolomics perspectives (Review). Int J Oncol 51: 5-17, 2017.

12. Yang W, Ma J, Zhou W, Cao B, Zhou X, Yang Z, Zhang H, Zhao Q, Fan D and Hong L: Molecular mechanisms and theranostic potential of miRNAs in drug resistance of gastric cancer. Expert Opin Ther Targets 21: 1063-1075, 2017.

13. Jeong SM, Xiao C, Finley LW, Lahusen T, Souza AL, Pierce K, Li YH, Wang X, Laurent G, German NJ, et al: SIRT4 has tumor-suppressive activity and regulates the cellular metabolic response to DNA damage by inhibiting mitochondrial glutamine metabolism. Cancer Cell 23: 450-463, 2013.

14. Moffat J, Grueneberg DA, Yang X, Kim SY, Kloepfer AM, Hinkle G, Piqani B, Eisenhaure TM, Luo B, Grenier JK, et al: A lentiviral RNAi library for human and mouse genes applied to an arrayed viral high-content screen. Cell 124: 1283-1298, 2006.

15. Livak KJ and Schmittgen TD: Analysis of relative gene expression data using real-time quantitative PCR and the $2^{-\Delta \Delta C \mathrm{~T}}$ method. Methods 25: 402-408, 2001.

16. Zhang Z, Li J, Zheng H, Yu C, Chen J, Liu Z, Li M, Zeng M, Zhou $\mathrm{F}$ and Song L: Expression and cytoplasmic localization of SAM68 is a significant and independent prognostic marker for renal cell carcinoma. Cancer Epidemiol Biomarkers Prev 18: 2685-2693, 2009.
17. Emerling BM, Weinberg F, Liu JL, Mak TW and Chandel NS: PTEN regulates p300-dependent hypoxia-inducible factor 1 transcriptional activity through Forkhead transcription factor 3a (FOXO3a). Proc Natl Acad Sci USA 105: 2622-2627, 2008.

18. Bell EL, Emerling BM, Ricoult SJ and Guarente L: SirT3 suppresses hypoxia inducible factor $1 \alpha$ and tumor growth by inhibiting mitochondrial ROS production. Oncogene 30: 2986-2996, 2011.

19. Finley LW, Carracedo A, Lee J, Souza A, Egia A, Zhang J, Teruya-Feldstein J, Moreira PI, Cardoso SM, Clish CB, et al: SIRT3 opposes reprogramming of cancer cell metabolism through HIF1 $\alpha$ destabilization. Cancer Cell 19: 416-428, 2011.

20. Adler V, Yin Z, Tew KD and Ronai Z: Role of redox potential and reactive oxygen species in stress signaling. Oncogene 18: 6104-6111, 1999.

21. Bell EL, Klimova TA, Eisenbart J, Schumacker PT and Chandel NS: Mitochondrial reactive oxygen species trigger hypoxia-inducible factor-dependent extension of the replicative life span during hypoxia. Mol Cell Biol 27: 5737-5745, 2007.

22. Chandel NS, McClintock DS, Feliciano CE, Wood TM, Melendez JA, Rodriguez AM and Schumacker PT: Reactive oxygen species generated at mitochondrial complex III stabilize hypoxia-inducible factor-1alpha during hypoxia: A mechanism of O2 sensing. J Biol Chem 275: 25130-25138, 2000.

23. Pore N, Jiang Z, Shu HK, Bernhard E, Kao GD and Maity A: Akt1 activation can augment hypoxia-inducible factor-1alpha expression by increasing protein translation through a mammalian target of rapamycin-independent pathway. Mol Cancer Res 4: 471-479, 2006.

24. Minet E, Arnould T, Michel G, Roland I, Mottet D, Raes M, Remacle $J$ and Michiels C: ERK activation upon hypoxia: Involvement in HIF-1 activation. FEBS Lett 468: 53-58, 2000.

25. Cassavaugh JM, Hale SA, Wellman TL, Howe AK, Wong C and Lounsbury KM: Negative regulation of HIF-1 $\alpha$ by an FBW7-mediated degradation pathway during hypoxia. J Cell Biochem 112: 3882-3890, 2011.

26. Lu K, Alcivar AL, Ma J, Foo TK, Zywea S, Mahdi A, Huo Y, Kensler TW, Gatza ML and Xia B: NRF2 induction supporting breast cancer cell survival is enabled by oxidative stress-induced DPP3-KEAP1 interaction. Cancer Res 77: 2881-2892, 2017.

27. Ngo HK, Kim DH, Cha YN, Na HK and Surh YJ: Nrf2 mutagenic activation drives hepatocarcinogenesis. Cancer Res 77: 4797-4808, 2017.

28. Abu-Alainin W, Gana T, Liloglou T, Olayanju A, Barrera LN, Ferguson R, Campbell F, Andrews T, Goldring C, Kitteringham N, et al: UHRF1 regulation of the Keap1-Nrf2 pathway in pancreatic cancer contributes to oncogenesis. J Pathol 238: 423-433, 2016.

29. Hayes AJ, Skouras C, Haugk B and Charnley RM: Keap1-Nrf2 signalling in pancreatic cancer. Int J Biochem Cell Biol 65: 288-299, 2015.

30. Sporn MB and Liby KT: NRF2 and cancer: The good, the bad and the importance of context. Nat Rev Cancer 12: 564-571, 2012.

31. Cardone RA, Casavola V and Reshkin SJ: The role of disturbed $\mathrm{pH}$ dynamics and the $\mathrm{Na}^{+} / \mathrm{H}^{+}$exchanger in metastasis. Nat Rev Cancer 5: 786-795, 2005.

32. Kato Y, Lambert CA, Colige AC, Mineur P, Noël A, Frankenne F, Foidart JM, Baba M, Hata R, Miyazaki K and Tsukuda M: Acidic extracellular $\mathrm{pH}$ induces matrix metalloproteinase-9 expression in mouse metastatic melanoma cells through the phospholipase D-mitogen-activated protein kinase signaling. J Biol Chem 280: 10938-10944, 2005.

33. Lu P, Takai K, Weaver VM and Werb Z: Extracellular matrix degradation and remodeling in development and disease. Cold Spring Harb Perspect Biol 3: a005058, 2011.

34. Yu J, Li J, Chen Y, Cao W, Lu Y, Yang J and Xing E: Snail enhances glycolysis in the epithelial-mesenchymal transition process by targeting FBP1 in gastric cancer. Cell Physiol Biochem 43: 31-38, 2017.

35. Yang MH, Wu MZ, Chiou SH, Chen PM, Chang SY, Liu CJ, Teng SC and Wu KJ: Direct regulation of TWIST by HIF-1alpha promotes metastasis. Nat Cell Biol 10: 295-305, 2008.

36. Arany Z, Foo SY, Ma Y, Ruas JL, Bommi-Reddy A, Girnun G, Cooper M, Laznik D, Chinsomboon J, Rangwala SM, et al: HIF-independent regulation of VEGF and angiogenesis by the transcriptional coactivator PGC-1alpha. Nature 451: 1008-1012, 2008. 